\title{
SALVAGE TREATMENT WITH GANCICLOVIR IN A SPLENECTOMIZED, POLYTRANSFUSED PATIENT AFFECTED BY SYSTEMIC INFLAMMATORY RESPONSE SYNDROME
}

\author{
D. TORRES, G. PARRINELLO, M. BELLANCA, R. MARRONE, F. CUTTITTA, G. VIRZI', \\ G. PIZZO, R. PETRANTONI and G. LICATA
}
Dipartimento Biomedico di Medicina Interna e Specialistica (Di.Bi.M.I.S.), Azienda Ospedaliera Universitaria Policlinico (A.O.U.P.) "Paolo Giaccone”, University of Palermo, Italy

Received November 17, 2013 - Accepted March 24, 2014

A 23-year-old man was admitted to our hospital with a 12-day history of daily fever. A clinical history revealed that 10 months previously, the patient had been splenectomized and polytransfused for a severe blunt trauma. His general clinical conditions rapidly worsened into a severe systemic inflammatory response syndrome. After 10 days of broad-spectrum antibiotic treatment, the temperature curve was unmodified and severe leukocytosis persisted $(44,300 \mathrm{ul})$ with absolute lymphocytosis. Laboratory tests ruled out hematological diseases, pneumonia, abscesses and endocarditis. In the light of IgM positivity for CMV (unconfirmed by PCR) and with the support of a PubMed search, we commenced a salvage treatment with intravenous ganciclovir, suspecting a viral infection or reactivation. After two days of therapy, an immediate defervescence was observed with a remarkable clinical improvement. After 10 days, the clinical syndrome had been completely resolved and the patient was discharged in good, general clinical health.

\section{Case report}

Prolonged fever is a medical challenge, especially when it is not responsive to empirical therapy and it rapidly evolves into a severe clinical picture, pending results from laboratory examinations (1). A 23-yearold man, previously in good health, was admitted with a 12 -day history of daily fever $\left(\mathrm{T} \max 39.3^{\circ} \mathrm{C}\right)$, prevalently serotin, preceded by chills, associated with myalgias and a cough. The fever was not responsive to home antibiotic therapy with amoxicillin/ clavulanic acid nor subsequently with ceftriaxone plus ciprofloxacin. A clinical history revealed that 10 months prior to this hospitalization, the patient had been splenectomized and polytransfused for a severe blunt trauma. He also reported fractures of the femur and patella which had undergone osteosynthesis. After the splenectomy, the patient had undergonet monthly antibiotic prophylaxis with diaminocillin for six months.

On admission, the patient was febrile but his clinical conditions were not compromised. Blood pressure was 140/75 $\mathrm{mmHg}$ and heart rate $114 \mathrm{bpm}$. A physical examination revealed laterocervical lymphadenopathy and no pathologic signs on the skin, abdomen, lungs or heart. Laboratory data revealed: white blood cells 33,230/ul (4,000$11,000 / \mathrm{ul})$, neutrophils $42 \%, 13,820 / \mathrm{ul} \quad(2,000-$ $8,000)$, lymphocytes $41 \%, 13,510 / \mathrm{ul}(1,000-5,000)$,

Key words: fever, splenectomed patient, leukocytosis, systemic inflammatory response syndrome, ganciclovir

Mailing address: Dr. Daniele Torres,

Dipartimento Biomedico,

di Medicina Interna e Specialistica (Di.Bi.M.I.S.)

0394-6320 (2014)

Azienda Ospedaliera Universitaria,

Policlinico (A.O.U.P.) "Paolo Giaccone",

Piazza delle Cliniche 2, 90127, Palermo, Italy

Tel.: +39333 2152198; Fax: +390916552198

e-mail: daniele_torres@libero.it DISCLOSURE: ALL AUTHORS REPORT NO CONFLICTS OF INTEREST RELEVANT TO THIS ARTICLE. 
Table I. Kinetic of patient's history and clinical evolution of illness.

Clinical event

Splenectomy and multiple trasfusions

Continous fever with Influenza-like symptoms

Significant leukocitosis, laboratory liver dysfunction

General clinical worsening, onset of facial/limb edema and coagulopaty

Positivity of serology for CMV, EBV and mycoplasma pneumoniae

Ganciclovir treatment

Fever lysis with remarkable clinical improvement

Thrombocytosis

Discharge
Time

Duration

10 months before hospitalization

12 days before hospitalization

23 days

On admission

4 days after hospitalization

8 days after hospitalization

10 days after hospitalization

2 days after Ganciclovir, 24 days after illness manifestation

4 days after Ganciclovir

10 days after Ganciclovir treatment

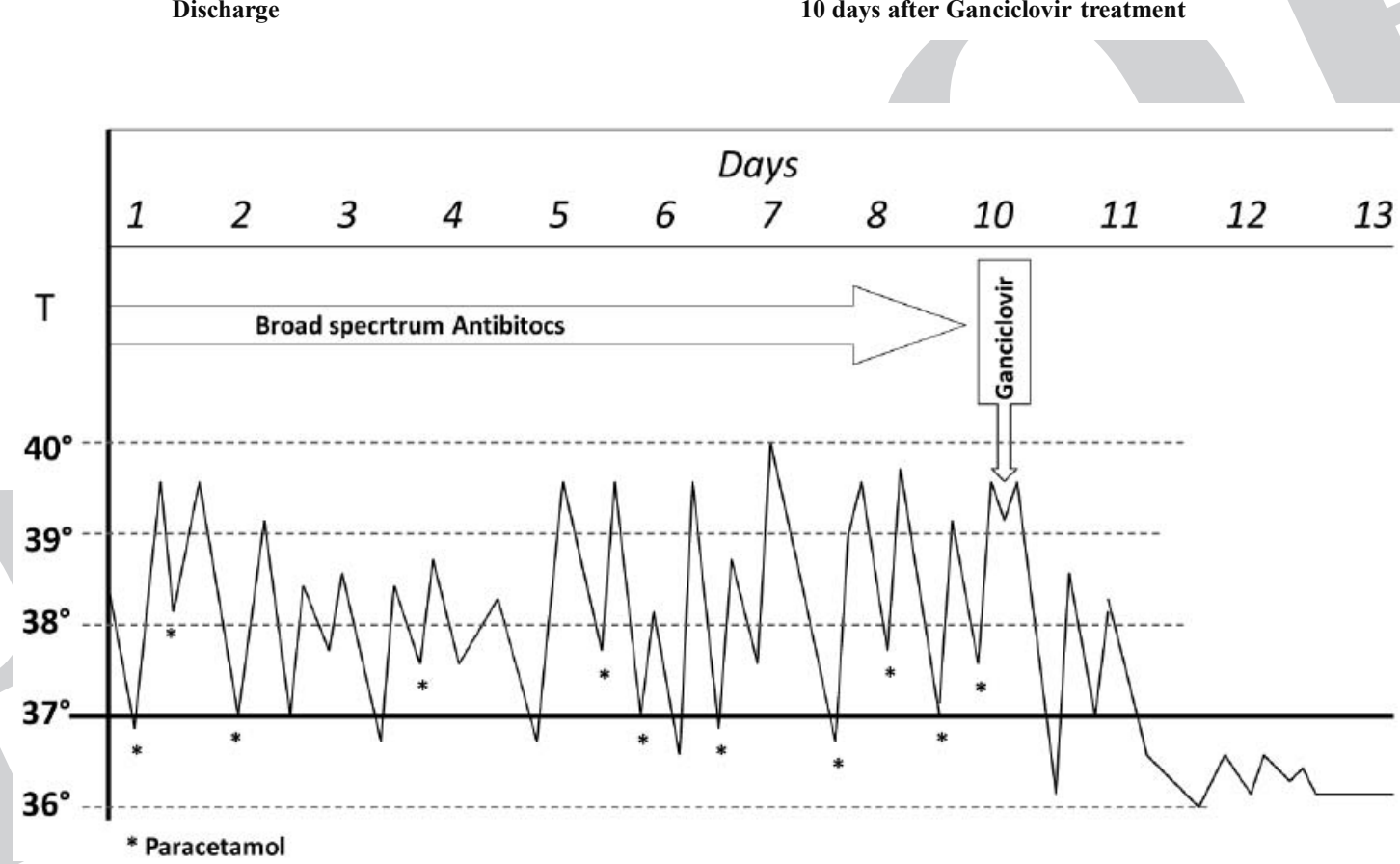

Fig. 1. Termic curve of patient and relation with treatment.

monocytes $11.2 \%, 3,730 / \mathrm{ul}(160-1,000)$, eosinophils $4.2 \%, 1,410 / \mathrm{ul}(0-800)$, basophils 2.3\%, 760/ul $(<200)$, platelets $394,000 / \mathrm{ul}(150,000-450,000 / \mathrm{ul})$; a hemoglobin level of $16.7 \mathrm{~g} / \mathrm{dl}(12-18 \mathrm{~g} / \mathrm{dl})$; albumin $3.5 \mathrm{~g} / \mathrm{dl}(3.4-4.8 \mathrm{~g} / \mathrm{dl})$; INR 1.3 (0.8-1.2); bilirubin $0.79 \mathrm{mg} / \mathrm{dl}(0.2-1.3 \mathrm{mg} / \mathrm{dl})$; alanine aminotransferase $129 \mathrm{U} / \mathrm{L}(<41 \mathrm{U} / \mathrm{L})$; aspartate aminotransferase 59 $\mathrm{U} / \mathrm{L}(<37 \mathrm{U} / \mathrm{L})$; gamma-glutamyltransferase 443 $\mathrm{U} / \mathrm{L}(<61 \mathrm{U} / \mathrm{L})$; alkaline phosphatase $762 \mathrm{U} / \mathrm{L}(<129$
$\mathrm{U} / \mathrm{L}) ; \mathrm{LDH} 887 \mathrm{U} / \mathrm{L}(240-480 \mathrm{U} / \mathrm{L})$; serum iron $25 \mathrm{mg} / \mathrm{dl}(59-158 \mathrm{mg} / \mathrm{dl})$; ferritin $393 \mathrm{ng} / \mathrm{ml}(30-$ $400 \mathrm{ng} / \mathrm{ml})$, and a CRP $4.69 \mathrm{mg} / \mathrm{dl}(<0.5 \mathrm{mg} / \mathrm{dl})$. A urinalysis was normal. A chest X-Ray excluded pneumonia, and abdominal ultrasounds showed hepatomegaly, various centimetric lymph nodes at the hepatic hilum and a small splenosis $(2 \mathrm{~cm})$ at the side of spleen. Considering this particular clinical picture, an empirical antibiotic treatment 
with teicoplanin, cefotaxime and claritromicin was immediately commenced. The diagnostic procedures for fever started and the pheripheral blood smear performed whilst suspecting a lymphoproliferative process, revealed: neutrophils 49\%, lymphocytes $37 \%$, monocytes $7 \%$, eosinophils $2 \%$, basophils $0 \%$ and $6 \%$ of large size lymphomonocytes with rare myeloid precursors. Beta2-microglobulin was 4.3 $\mathrm{mg} / \mathrm{dl}(0.8-2.2 \mathrm{mg} / \mathrm{dl})$.

After 5 days of hospitalization, a high and continuous fever persisted (Fig. 1) and the general clinical conditions rapidly worsened into a severe systemic inflammatory response syndrome (SIRS), which was complicated by facial and limb edema and associated with: proteinuria (388 $\mathrm{mg} / 24 \mathrm{hrs}$, n.v. $<140)$, hypocalcaemia $(7.1 \mathrm{mg} /$ dl, n.v. 8.4-10.2), hypoproteinemia (5.2 $\mathrm{mg} / \mathrm{dl}$, n.v.6.6-8.7), hypertriglyceridemia (243 $\mathrm{mg} / \mathrm{dl}$, n.v. <200), hyponatremia (125 mEq/L, n.v.132147), hypoalbuminemia (2.2 mg/dl, n.v.3.4-4.8), hypofibrinogaenemia (112 mg/dl, n.v.150-450), low AT III (61\%, n.v.80-120) and Pches reduction (3040 U/L, n.v.5320-12920), mild PTT prolongation (39 sec, n.v.24-36), and high levels of D-dimer (3059 ng/ $\mathrm{ml}, \mathrm{n.v} .<280$ ) with normal total bilirubin and platelet count. On the basis of this result, a substitutive treatment with Antitrombin III was commenced. Doppler echocardiography excluded endocarditis and total body CT with contrast showed no sources of infection or abdominal-thoracic limphoadenopathy. To exclude a bone infection, orthopantomography and X-rays of femur and knee were also carried out and a small focus of osteomyelitis of alveolar bone of teeth 37 and 38 was revealed.

Considering the unsuccessful antibiotic therapy, treatment was therefore shifted to metronidazole, piperacillin/tazobactam, levofloxacinand daptomicin. Meanwhile, all the results of laboratory examinations had been received. Septifast test, repeated blood, urine, and sputum cultures as well as throat swab and Mantoux test were all negative. Serology for main viral and bacterial infections (including H1N1, HIV, hepatitis A, B, C, and D virus, Adenovirus, Herpes Simplex virus, Brucella, Rickettsia, Leishmania, Chlamydia, Salmonella and Toxoplasma gondii, QuantiFERON-TB) revealed only: IgM positivity for Epstein-Barr virus VCA and $\mathrm{IgM} / \mathrm{IgG}$ positivity for Mycoplasma Pneumoniae (IgM 38.6 U/ml - n.v.
<17; IgG $278 \mathrm{U} / \mathrm{ml}$-v.n. <30) and Cytomegalovirus (IgM $11.9 \mathrm{U} / \mathrm{ml}$ - n.v. <1.1; IgG $14.6 \mathrm{U} / \mathrm{ml}$-n.v. $<1.1)$. CMV infection was not confirmed by CMV protein pp65 antigenemia and CMV DNA by PCR and, for this reason, a probable cross-reactivity was considered. A fundus oculi examination also ruled out retinitis. Complete autoanticorpal screening showed a positivity of ANA at 1:160 title with a granular pattern.

After 10 days of hospitalization, the temperature curve was unmodified despite treatment and was poorly responsive to paracetamol (Fig. 1). Leukocytosis persisted at very high levels $(44,300 \mathrm{ul})$ with absolute lymphocytosis $(19,700 /$ ul), monocytocis $(7,100 / \mathrm{ul})$ basophilia (800/ul), mild neutrophilia $(10,400 / \mathrm{ul})$ and the patient's general conditions became extremely critical. Repeated pheripheral blood smears showed $8 \%$ of myeloid and lymphomonocyte precursors. Flow cytometry revealed $63 \%$ of lymphocytes $(25,489$ on 40,460 of WBC) with a mild expansion of the T-lymphocyte population and an inversion of the $\mathrm{CD} 4+/ \mathrm{CD} 8+$ ratio. Therefore, with a view to conducting an osteomedullary biopsy to exclude a lymphoproliferative disorder or hemophagocytic syndrome, all clinical data and history were carefully re-reviewed by the medical team. We considered as very significant the following clinical elements: splenectomy, fever, lymphocytosis and positivity for CMV, EBV and Mycoplasma pneumoniae.

Thus, a search was conducted on PubMed using the following strings: i. splenectomy/fever/ lymphocytosis; ii. Splenectomy/fever/Epstein-Barr virus; iii. splenectomy/fever/cytomegalovirus; and iv. splenectomy/fever/Mycoplasma Pneumoniae. The only relevant result, obtained using both the first and third strings, was the following: "Severe cytomegalovirus infection in multiply transfused, splenectomized, trauma patients" (2). In light of this search, a result compatible with the clinical history (splenectomy, multiple blood transfusions, severe febrile illness), and considering the aggressiveness of the clinical picture, a salvage treatment with intravenous ganciclovir ( $5 \mathrm{mg} / \mathrm{kg}$ every $12 \mathrm{~h}$ ) was commenced whilst suspecting a viral infection or reactivation. After two days of therapy, an immediate defervescence was observed and the patient presented a remarkable clinical improvement (Fig. 1). 
After 4 days, a new occurrence of significant thrombocytosis ( $\max$ value $813,000 / \mathrm{ul}$ ) was also registered. After 5 days of therapy, the patient reported subjective well-being and leukocytosis was considerably reduced $(28,870 v s 44,300)$ with a normalization of the neutrophil cell count and a reduction in lymphocytosis. After 10 days, treatment with ganciclovir was discontinued, and the patient was discharged in a good, clinical general health. At 1-month and 1-year clinical follow-up the patient was found to be well.

This unusual case illustrates the presentation of a viral infection in a splenectomized, immunocompetent patient as a high continuous fever with sustained leukocytosis and lymphocytosis; it also describes its clinical evolution as a severe SIRS with liver dysfunction. Table I summarizes the kinetic and evolution of the illness.

Commonly, severe viral infections manifest themselves in immunocompromized patients, such as HIV patients, transplant allograft recipients, patients taking immunosuppressive agents or those with hematological diseases (leukemia and lymphoma), and they are frequently due to CMV (3). This report confirms the possibility that the spleen has a role not only in preventing bacterial sepsis with encapsulated organisms but also in providings first line filtration and a defense against the virus (4). Consequently, splenectomy modifies the pattern of viral infection in immunocompetent subjects and increases its severity, also leading to a quite aggressive disease.

Some authors have described that patients splenectomized for trauma have significantly decreased levels of IgM while levels of $\operatorname{IgA}$ and $\operatorname{IgG}$ are significantly increased (5). IgM synthesis by peripheral blood mononuclear cells in splenectomized patients was markedly diminished in vitro but IgG synthesis was normal. The splenectomized population has shown a total lymphocyte count with a significantly greater number of $\mathrm{T}$ cells and $\mathrm{B}$ lymphocytes than in the control group. Characterizing the mechanism responsible for the altered immune response in splenectomized patients, in vitro experiments have revealed an impaired T-helper cell capacity in addition to an intrinsic B-cell defect. The proliferative responses of lymphocytes to phytohemagglutinin is diminished in splenectomized patients (6). The significantly higher
B-lymphocyte counts of splenectomized patients may be due to loss of the reservoir function of the spleen. A splenectomy induces a change in the lymphocyte recirculating pool due to the loss of an important anatomical site of migration. However, some authors have observed that the percentages of total T-cells (CD3), T-helper cells (CD4), and T-suppressor cells (CD8) are reduced in all splenectomized subjects, but the results are statistically significant only for CD4 cells (7). Conversely, peripheral CD8+ population showed an increase in splenectomized subjects since its percentage in the spleen is normally high. Consequently, the ratio of CD4/CD8 cells is significantly decreased in splenectomized subjects (8).

The level of the total complement $\mathrm{CH} 50$ in splenectomized population is significantly less than in the control group (5). Other authors have demonstrated that levels of $\mathrm{C} 3, \mathrm{C} 4$, and $\mathrm{C} 5$ are similar in splenectomized population and controls (6). These data demonstrate persistent abnormalities in the immune function in adult asplenic trauma patients in the absence of underlying lymphoreticular disorders, therefore suggesting a possible explanation for an increased septic risk in this patient group.

Considerable circumstantial data strongly suggest that the virus involved in primary infection and reactivation or reinfection after transfusion is that of CMV. The incidence of these infections appears related both to the number of donors and to the quantity of blood received by a patient. CMV infection following transfusion is most likely secondary to the reactivation of the latent virus either in donor white blood cells or host tissues. Most CMV infections acquired after transfusion are either asymptomatic or characterized by a selflimited infectious mononucleosis syndrome. There are, however, specific groups of patients for whom a primary CMV infection after transfusion may cause significant morbidity and/or mortality. The patients at risk are seronegative and they include: pregnant women, premature infants, recipients of organ transplants from seronegative donors, and limited groups of severely immunosuppressed oncology patients.

Seropositive individuals have latent CMV infection with viral DNA present in peripheral blood leucocytes. Calamy (11) reported that a CMV 
infection is most frequently observed after open heart surgery with extracorporeal circulation, common after thoracic surgery without extracorporeal circulation, and after splenectomies, although very rarely after other types of surgery. Fresh blood was initially suspected of being the cause, but later investigators found no difference between fresh and preserved blood. Seroconversion and/or infection risks can be sharply cut by using deleukocyted blood or frozen deglycerolized erythrocytes, both of which are free of viable leukocytes. Evidence, obtained from a number of reports, suggests that the provision of leucodepleted blood components may be as effective as the use of components from CMV-seronegative donors in preventing CMV infection and disease. Seroconversion risk increases significantly when CMV antibodies are present in transfused blood. However, the presence of these antibodies in the recipient's blood appears to afford protection against seroconversion and/or infection, except in the case of immunodepressed patients. Calamy concluded that CMV febrile mononucleosis syndrome might be due to latent CMV in the transfused blood. This transfusion of allogenic blood might induce an immunological reaction that reactivates the latent CMV transmitted to recipient (11). Transfusionacquired CMV mononucleosis after a trauma-related splenectomy may cause prominent lymphocytosis and constitute a distinct clinicopathologic syndrome, which can occur early or after decades (6).

Baumgartner et al. (2) have reported increased severity and morbidity from CMV infection after splenectomy. In particular, throughout a 2-5-year period, previously healthy young men who had undergone a splenectomy and received multiple transfusions for trauma had a severe CMV infection. Their illness was characterized by a long period of high fever, severe interstitial pneumonitis with dyspnea and hypoxemia, and an unusually high lymphocytosis (12 000-26 000 cells/microliter) with numerous atypical forms. In one case, widespread CMV pneumonitis was confirmed at necropsy. Two other reports described fatal CMV infection in splenectomized patients $(9,10)$.

In light of our report, we cannot exclude that a viral infection other than CMV may be responsible for the same clinicopathologic syndrome in splenectomized polytransfused patients. Thus, for the practicing clinician, recognizing these features may aid timely diagnosis.

To conclude, this report suggests that the clinical history and timing are important features which may lead to the correct diagnosis and effective treatment also when the illness is aggressive and that laboratory results are not conclusive. Finally, PubMed is a very useful tool with which to confront an uncommon clinical picture.

\section{REFERENCES}

1. Mourad O, Palda V, Detsky A. A comprehensive evidence-based approach to fever of unknown origin. Arch Intern Med 2003; 163:545-51.

2. Baumgartner JD, Glauser MP, Burgo-BlackAL, Black RD, Pyndiah N, Chiolero R. Severe cytomegalovirus infection in multiply transfused, splenectomized, trauma patients. Lancet 1982; 2(8289):63-6.

3. Malangoni MA, Dillon LD, Klamer TW, Condon RE. Factors influencing the risk of early and late serious infection in adults after splenectomy for trauma. Surgery 1984; 96(4):775-83.

4. García-Erce JA, García-Foncillas R, Grasa-Ullrich JM, Giralt M. Fatal cytomegalovirus infection in a patient after splenectomy and multiple transfusions following trauma. Med Clin (Barc) 2002; 118(8):318.

5. Kukoc M, Sapunar A, Vidjak V, Aras N, Grandić L, Radonić V, Matijević M. Permanent decrease in immune function in patients with splenectomy for trauma. Acta Chir Iugosl 1989; 36(S):495-9

6. Downey EC, Shackford SR, Fridlund PH, Ninnemann JL. Long-term depressed immune function in patients splenectomized for trauma. J Trauma 1987; 27(6):661-3.

7. Wolf HM, Eibl MM, Georgi E, Samstag A, Spatz $M$, Uranüs S, Passl R. Long-term decrease of CD4+CD45RA+ T cells and impaired primary immune response after post-traumatic splenectomy. $\mathrm{Br}$ J Haematol 1999; 107(1):55-68.

8. Tsai MC, Lin SL, Chuang CY. Changes in T-lymphocyte subpopulations in patients splenectomized for trauma. J Formos Med Assoc 1991; 90(3):240-3.

9. Han XY, Hellerstedt BA, Koller CA. Postsplenectomy cytomegalovirus mononucleosis is a distinct clinicopathologic syndrome. Am J Med Sci 2010; 
339(4):395-9.

10. de Górgolas Hernández-Mora M, Jiménez Moreno A, Fernández Guerrero ML, Gadea Gironés I. Fatal infection by cytomegalovirus in a patient after splenectomy and transfusion following trauma. Med
Clin (Barc) 2001; 116(11):439

11. Calamy G, Mille C. The role of transfusions in postoperative cytomegalovirus infections (in non-immunosuppressed subjects). Rev Fr Transfus Immunohematol 1984; 27:355-64. 